\title{
Téoros
}

Revue de recherche en tourisme

\section{Installations olympiques, régénération urbaine et tourisme}

\section{Jean-Pierre Augustin}

Volume 27, numéro 2, été 2008

Les grands équipements touristiques

URI : https://id.erudit.org/iderudit/1070795ar

DOI : https://doi.org/10.7202/1070795ar

Aller au sommaire du numéro

Éditeur(s)

Université du Québec à Montréal

ISSN

0712-8657 (imprimé)

1923-2705 (numérique)

Découvrir la revue

Citer cet article

Augustin, J.-P. (2008). Installations olympiques, régénération urbaine et tourisme. Téoros, 27(2), 31-35. https://doi.org/10.7202/1070795ar d'utilisation que vous pouvez consulter en ligne.

https://apropos.erudit.org/fr/usagers/politique-dutilisation/ 


\section{Installations olympiques, régénération urbaine et tourisme}

\section{Jean-Pierre Augustin}

Les villes et le tourisme ont toujours été liés et la tendance s'accentue avec la multiplication d'équipements urbains attractifs et d'événements multiples qui créent de fortes synergies avec le loisir, le sport et la culture. Parmi ces événements, les Jeux olympiques (JO) offrent aux villes choisies une occasion exceptionnelle de valoriser leur audience et leur attractivité. Les JO nécessitent la construction d'infrastructures d'accueil, de transports et de communication qui participent largement aux transformations urbaines en présentant deux caractéristiques d'aménagement. La première est liée à l'engagement de respecter un calendrier précis pour la réalisation des projets retenus. En effet, les villes obtiennent les jeux comme résultat de la présentation de leur candidature ; une fois désignées par les instances officielles, elles sont contraintes d'édifier les équipements proposés. La seconde concerne l'élargissement du programme sportif en projet de ville ou en projet urbain. Ces événements qui rassemblent des centaines de milliers de spectateurs et qui intéressent des centaines de millions d'auditeurs et de téléspectateurs (de 2 à 4 milliards pour les Jeux olympiques et les mondiaux de football) deviennent un prétexte pour accélérer les mutations urbaines (Gold et Gold, 2007) et valoriser l'image de la ville inscrite dans une compétition internationale. L'édification de grands sites olympiques amène à réfléchir aux recompositions urbanistiques qu'ils favorisent et aux effets d'entraînement qu'ils suscitent sur le plan du tourisme international (Augustin, 2007).

\section{Tendances urbanistiques et sites olympiques}

L'inscription des grands sites sportifs dans l'espace des villes depuis le début du $X X$ siècle ne peut pas être dissociée de I'histoire et des tendances de l'urbanisme marquées par plusieurs courants de pensée. L'idée de fixer des activités humaines dans des espaces appropriés est ancienne, mais elle s'est largement renforcée avec l'émergence de la ville industrielle. Les théoriciens fonctionnalistes proposent de délimiter les unités spatiales de base pour les activités industrielles, l'habitat des travailleurs et les équipements d'accompagnement. De Tony Garnier (1869-1948) à Le Corbusier (18871965), de nombreux urbanistes généralisent cette conception du traitement de l'espace avant qu'une politique publique de l'urbanisme favorisant la concertation entre l'État et les collectivités locales ne s'instaure. Des directives urbaines s'imposent dès 1950 en se référant aux fonctions définies par les congrès internationaux d'architecture moderne (CIAM) dans le courant des années 1930 : habiter, travailler, circuler, se recréer. Les nouveaux territoires urbains s'ordonnent dans le cadre d'un zonage qui redéfinit les attributions de l'espace et s'établissent généralement dans les secteurs libres ou périphériques des villes. Ces tendances urbanistiques sont largement internationales et ont dans le domaine des sites sportifs favorisé l'édification d'équipements adaptés aux différents niveaux d'espace, de l'unité de voisinage à l'agglomération, mais aussi la construction d'ensembles spécifiques de grande taille dans ou à la périphérie des villes.

Or il apparaît aujourd'hui manifeste que le modèle fonctionnel favorisant un zonage trop strict et rationnel de l'espace est remis en question, non seulement dans ses réalisations, mais dans son principe même. Si l'organisation des villes nécessite à l'évidence des aménagements fonctionnels, une conception systématique visant à enfermer les hommes et les activités dans un maillage territorial prédéfini s'est révélée inopérante et de surcroît dangereuse. L'évolution urbaine en raison des transformations économiques et des mutations sociales a progressivement entraîné la modification de ces normes. Des recherches et des expérimentations multiples se sont succédé depuis une trentaine d'années et posent la question du renouvellement des conceptions relatives au niveau d'intégration des équipements. Ainsi, la notion de projet urbain s'est constituée comme une alternative à l'urbanisme fonctionnaliste dans le courant des années 1970 (Devillers, 1994). La démarche du projet traite de l'aménagement en termes concrets, en s'intéressant à la forme, à la dimension des lieux et à l'intégration des équipements dans la ville. L'espace dont il est question n'est pas l'espace en général, mais un espace particulier, inscrit dans l'histoire, dont il faut tenir compte (Huet, 1993).

Les tendances urbanistiques ne se limitent cependant pas à l'opposition entre un urbanisme de zone et un urbanisme de continuité ; d'autres éléments, concernant directement les édifices sportifs, interfèrent fortement. Parmi ceux-là, il faut mentionner l'urbanisme de réseaux tenant compte des mobilités des flux et des communications et l'urbanisme d'image correspondant à l'accroissement de la compétition entre les métropoles urbaines. Une autre tendance, non sans effet sur les conceptions des grands sites sportifs, est liée à la concurrence qui se joue entre les grandes 
villes des pays développés. L'élargissement du monde et la compétition internationale ont favorisé les initiatives entrepreneuriales des grandes métropoles. Le marketing urbain et l'urbanisme de communication amènent les villes à promouvoir des sites sportifs s'inscrivant dans des opérations d'architecture et d'urbanisme de prestige. Les dernières villes olympiques retenues (Séoul 1988, Barcelone 1992, Atlanta 1996, Sydney 2000, Athènes 2004, Pékin 2008, Londres 2012) confirment ces tendances. Dans le cas de Barcelone, l'objectif affirmé du projet de ville est bien de se placer dans le peloton de tête des grandes métropoles européennes.

II ne s'agit pas ici de proposer un inventaire, mais de montrer, à partir de quelques exemples, comment les sites olympiques s'inscrivent dans des projets de villes et comment les infrastructures et les équipements sportifs renforcent l'offre touristique des villes. Pour cela, trois sites ont été retenus. Le premier, celui du Parc de Montréal en 1976, révèle qu'un projet fonctionnel peut servir, a posteriori, de point de départ à un projet urbain touristico-sportif. Dans le deuxième, celui du site de Barcelone en 1992, il s'agit d'un projet urbain qui se transforme en projet d'eurocité touristique. Enfin, dans celui de Pékin en 2008, il s'agit à la fois de réaménager la vieille ville et de l'ouvrir à la modernité et au tourisme international. Ainsi, sur une période de 32 ans, de 1976 à 2008, et d'autres exemples pourraient être évoqués, on assiste bien, lors des JO, à l'accélération des aménagements urbains et des installations sportives qui favorisent l'attractivité touristique.

\section{Montréal 1976 et l'affirma- tion de la métropole franco- canadienne}

C'est en 1970 que Montréal se voit confiée par le Comité international olympique (CIO) l'organisation des Jeux d'été de 1976. Après Saint-Louis en 1904 et Los Angeles en 1932, la métropole québécoise devient la troisième ville organisatrice des JO en Amérique du Nord. Ce choix s'inscrit dans le contexte de la Révolution tranquille, une période de mutation débutant en 1960 et visant à moderniser la province francophone du Québec. Montréal cherche à s'affirmer comme la métropole franco-canadienne, tout en visant à maintenir son statut de capitale culturelle

et économique du pays. Pour cela, elle s'engage dans la valorisation de grands événements internationaux, l'Exposition universelle en 1967, l'exposition Floralies en 1973 et enfin les Jeux olympiques en 1976. Ces événements sont dus à la détermination du maire Jean Drapeau qui, en exerçant un large contrôle sur le corps politique local et l'appareil municipal, a réussi à les imposer, malgré l'opposition de mouvements communau-

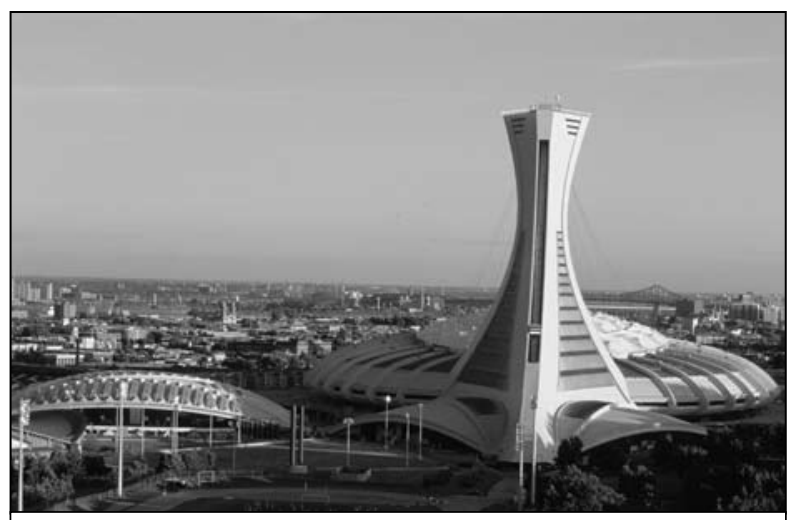

Illustration 1 : Stade olympique de Montréal. Photo : Linda Turgeon, Tourisme Québec. taires actifs dans les quartiers

populaires. Deux périodes vont se succéder, celle de la mise en œuvre du Parc olympique symbolisé par le stade, puis après le changement de municipalité à Montréal, la tentative, a posteriori, de réaliser un projet urbain à partir du nouveau pôle touristico-sportif.

Le complexe olympique s'établit à la limite du quartier Maisonneuve dans un espace de 55 hectares où sont prévus un stade, un centre aquatique, un vélodrome et le village. Ce dernier, nommé "Pyramides olympiques ", est composé de deux édifices jumeaux de 20 étages destinés à loger les athlètes. L'ensemble correspond à un projet sans liens avec les quartiers environnants et le stade conçu par l'architecte français Roger Taillibert se fonde sur l'idée de l'exploit architectural. II s'agit d'une structure de béton articulée sur 34 consoles auxquelles sont accrochés les gradins. La plus haute tour penchée du monde domine le stade et sert d'appui à 26 câbles de suspension qui retiennent le toit mobile susceptible de se déployer comme un parachute au-dessus du stade. Le Vélodrome épouse la forme du casque que portent les cyclistes grâce à un toit festonné d'un diamètre de 160 mètres et reposant sur quatre butées.

Original sur le plan architectural, le complexe olympique laisse en héritage une dette publique nécessitant plusieurs décennies de remboursement. II a été dès son origine et reste encore le projet le plus controversé de la ville. Le stade, qui peut accueillir 56000 personnes assises, a de la difficulté à trouver un tel public et est utilisé pour des concerts de rock, des opéras, des manifestations religieuses et politiques. La tour penchée est une des attractions principales de la ville ; la salle d'observation permet une vision panoramique de l'agglomération, facilitée par l'installation d'un centre d'interprétation. Le village devient un ensemble résidentiel et commercial, même si les galeries et les passages extérieurs sont peu adaptés aux hivers de Montréal. Le Vélodrome, en raison du peu d'intérêt du public pour le cyclisme en salle, a été reconverti en musée vivant de l'environnement et des sciences naturelles. Appelé Biodôme, il reproduit, à travers quatre écosystèmes, les milieux de la jungle amazonienne, du Saint-Laurent, de la forêt laurentienne et du monde polaire.

Si la reconversion du parc olympique en lieu d'attraction touristico-sportif a été en partie réussie, il a fallu attendre la fin des années 1980 pour que la nouvelle municipalité, avec le soutien du « rassemblement des citoyens et citoyennes de Montréal ", envisage d'utiliser cet ensemble fonctionnel comme support d'un projet urbain et touristique (Ville de Montréal, 1991). Partant de l'idée que les équipements du Parc olympique et du Jardin botanique n'ont pas eu d'effets sur le secteur puisqu'ils ont été conçus pour eux-mêmes, le projet propose de valoriser le patrimoine architectural et de soutenir des activités économiques afin d'intégrer les quartiers proches dans la dynamique d'attraction du pôle et de les faire bénéficier de ses retombées économiques et sociales. Préparé par le Bureau du plan d'urbanisme de la Ville de Montréal en 1991, le premier volet du projet concentre les efforts autour de l'axe Morgan, limité par le parc du même nom et 
celui de Genevilliers, où plusieurs bâtiments, comme le marché et les bains publics, mais aussi l'école Maisonneuve (1890), le théâtre Granada (1928) et les banques Molson (1906) et Toronto (1911), représentent un groupe d'intérêt patrimonial.

Le second volet propose le soutien d'une fonction industrielle dans le quartier, dont certains bâtiments comme celui de la compagnie Johnson (1904) ont été restaurés. II s'appuie sur les corporations de développement économique et communautaire (CDEC) et envisage une série d'actions dans le cadre d'une politique publique municipale avec, notamment, l'élaboration de plans d'aménagement intégrés et la mise sur pied de centres d'aide aux entreprises. L'ensemble des atouts patrimoniaux et industriels est complété par la rénovation des artères et des nœuds urbains. En permettant l'aménagement paysager de l'ensemble, la valorisation de la composante muséologique, la mise en place d'animations et de circuits touristiques, le projet vise à exploiter l'attraction des équipements olympiques pour intéresser les visiteurs de l'agglomération et les touristes étrangers.

Ainsi, le Parc olympique de Montréal, conçu dans une perspective fonctionnelle et architecturale, se voit complété par un projet urbain qui tente, a posteriori, d'utiliser la dynamique d'attraction du site. À Barcelone, c'est l'inverse qui se produit, puisque les projets urbains sont en partie délaissés au profit de la logique de modernisation de la ville.

\section{Barcelone 1992, les JO et la modernisation d'une eurocité touristique}

Le choix de Barcelone comme ville olympique pour les jeux de 1992 est décidé en 1986, contre Paris, à partir d'une argumentation fondée sur l'existence d'équipements et sur un ambitieux projet urbain de restructuration. Les jeux sont l'occasion d'une modernisation qui bouleverse la ville en favorisant l'expansion économique et la mutation du tissu social.

Comme dans le cas de Montréal dans les années 1970, le contexte politique de Barcelone dans les années 1980 est décisif sur les orientations choisies. En 1979, les premières élections municipales libres amènent au pouvoir une majorité de gauche qui propose une politique urbaine de concertation s'inscrivant délibérément dans la perspective d'un projet urbain qui valorise les aménagements de proximité en accord avec les associations d'habitants. II s'agit notamment de repenser les prolongements du plan en damiers conçu en 1859 par l'architecte Cerda. L'effervescence urbanistique de secteur proposée à Barcelone est présentée dans de multiples articles et publications qui affirment la volonté de transformations urbaines à partir de " ces creux ", en assurant l'attractivité touristique, comme le note l'architecte Oriol Bohigas.

La préparation des JO est l'occasion de lancer les grands travaux de modernisation concernant les communications et les infrastructures urbanistiques (Seguillinas, 1993). La création d'un anneau périphérique en partie souterrain permettant de relier les sites olympiques, le réaménagement du réseau ferroviaire et des gares, la réorganisation de l'aéroport avec de nouveaux terminaux et une liaison par train à la ville transforment les modes de communication. Ces travaux sont complétés par la construction de la tour de télécommunications de Collserolo, qui s'élève à 268 mètres. Les infrastructures urbanistiques sont concentrées sur quatre lieux.

Le site principal est celui de la colline de Montjuic, qui fut choisie en 1929 comme lieu de l'exposition universelle et où fut édifié le stade de l'olympiade populaire de 1936. Réhabilité par l'architecte italien Gregotti, ce stade peut recevoir 60000 personnes pour les cérémonies d'ouverture et de fermeture des jeux. À proximité se situe le palais omnisports de Sant-Jordi, conçu par le Japonais Isozaki. Un large parvis conduit aux portiques néoclassiques proposés par Boffil pour l'université des sports. La piscine, les terrains de hockey et de baseball, les pistes d'entraînement et divers équipements complètent ce vaste ensemble qui est prolongé par le nouveau parc du Midi, comprenant un jardin botanique et un auditorium de 100000 places. Le deuxième site, celui du village olympique, est l'occasion d'une importante opération de rénovation littorale qui entraîne la destruction d'un quartier ouvrier pour ouvrir la ville sur la mer.

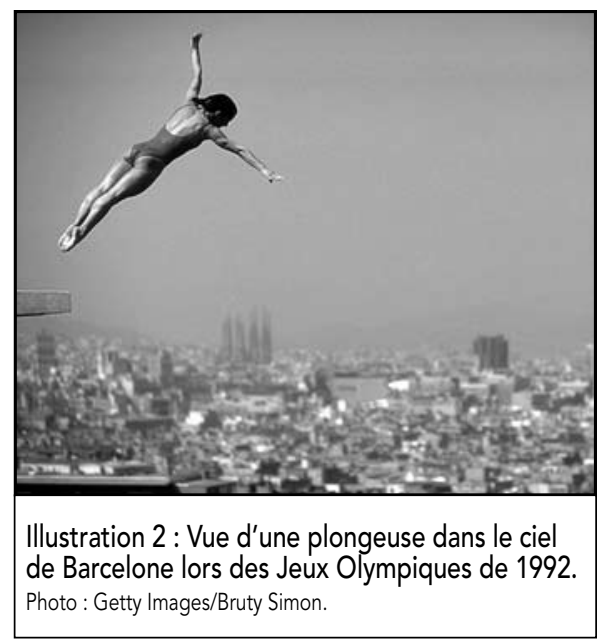

À sa place et dans son prolongement, un nouveau quartier résidentiel est édifié à côté d'un parc du littoral gagné sur les emprises ferroviaires et protégé des contraintes de la voie rapide enterrée sur presque tout son parcours. Deux mille logements et deux tours de 44 étages, l'une destinée à un hôtel de luxe et l'autre à des bureaux, deviennent les bâtiments phares du quartier. Le Palais des congrès, le nouveau port de plaisance avec 743 anneaux et, surtout, le parc de 50 hectares complètent l'ensemble.

À l'aplomb de la montagne, au nord de Barcelone, le troisième site olympique est celui du Val d'Hébron. Ce secteur a longtemps été une zone résidentielle avant que la spéculation immobilière des années 1960 n'y élève des barres et des tours d'habitation dans le cadre d'un vaste projet interrompu par la crise des années 1970. La vocation sportive de ce secteur débute avec la construction en 1984 du vélodrome de 6500 places pour le championnat du monde de cyclisme. Les architectes barcelonais complètent le quartier en créant les installations sportives pour le tir à l'arc, le volleyball et le tennis, ainsi que des habitations et un bâtiment central réservés à la presse et aux arbitres.

Enfin, le quatrième site se situe à l'extrémité ouest de la diagonale dans une zone limitrophe des trois communes de Barcelone, l'Hospitalet et l'Espluges. Une opération de rénovation urbaine permet de désenclaver un secteur de 30 hectares et de réaliser un nouveau quartier à proximité du stade de 120000 places du Nou Camp, fief du 


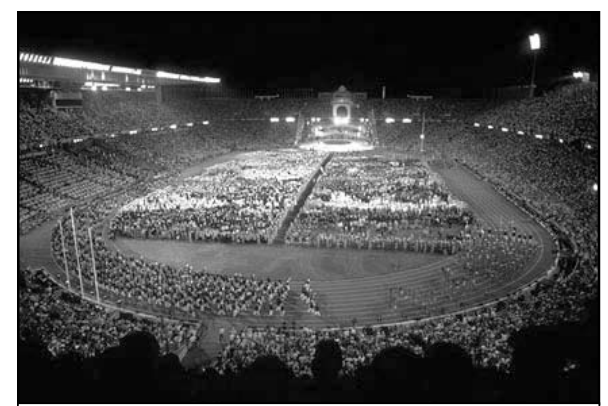

Illustration 3 : Stade olympique des Jeux de Barcelone (1992).

Photo : ClO/Collection du Musée Olympique.

Barça, un des grands clubs européens de football ; une ville équestre est édifiée, renforçant le caractère sportif de l'ensemble.

Au total, la démarche urbanistique mise en œuvre à Barcelone est exemplaire de la volonté de mêler, dans la mesure du possible, projet de ville et projet urbain (Sobry, 1993). Au choix de redonner leurs valeurs aux espaces publics et à tout ce qui lie les bâtiments, les quartiers et les fonctions urbaines, s'ajoute l'édification de monuments symboles s'inscrivant dans un urbanisme d'images. Les aménagements urbains de Barcelone à l'occasion des $\mathrm{JO}$ accompagnent la revalorisation d'une ville engagée dans la compétition internationale et cherchant à s'affirmer comme capitale du Sud en augmentant son attractivité touristique.

\section{Pékin 2008, s'affirmer comme une ville mondiale ouverte au tourisme}

Pour les jeux de 2008, la dernière phase, celle du vote, se jouée à Moscou en juillet 2001, lors de la $112^{\mathrm{e}}$ session du ClO, entre Osaka, Toronto, Istanbul, Paris et Pékin, qui a été sacrée ville olympique. Le choix souligne l'ouverture de la Chine et engage sa capitale politique dans un immense chantier de rénovation urbaine. Les JO de Pékin veulent marquer l'entrée véritable de la Chine dans le club des grandes puissances et souligner les nouveaux équilibres du monde qui tournent la page à ceux d'hier, organisés par la triade autour des ÉtatsUnis, de l'Europe et du Japon. Les JO sont l'occasion de présenter la vitrine de la Chine moderne en proposant la vision d'un pays stable et prospère, uni, à l'image du slogan choisi : "Bienvenue à Pékin, un seul monde, un seul rêve. " Pour cela, la mobilisation des acteurs politiques et économiques est centrée sur trois objectifs majeurs : le remodelage urbain, l'amélioration des réseaux de transport et l'attractivité touristique. Pékin est devenu un immense chantier d'urbanisme qui s'organise autour des cinq infrastructures emblématiques qui veulent en faire une capitale de l'architecture d'avant-garde.

Le stade olympique, baptisé « nid d'oiseau » en raison de ses poutres d'acier et de béton entrelacées, est dessiné par les Suisses Herzog et Meuron pour accueillir 91000 spectateurs. La piscine olympique, appelée " cube d'eau ", est une sorte de bulle cubique proposée par les Australiens, concepteurs du stade olympique de Sydney, pour recevoir 10000 personnes. Les deux tours consacrées aux médias et à la télévision (CCTV), qui semblent se tordre à leur sommet pour s'incliner vers la terre, sont présentées par le Néerlandais Rem Koolhas et l'Allemand Ole Scheeren comme une révolution en termes d'immeuble de bureaux. Le nouvel aéroport, conçu par l'Anglais Norman Forster (déjà architecte de l'aéroport de Hong Kong), est pensé pour accueillir 46 millions de passagers par an. Enfin, l'opéra ou grand théâtre national de Chine, œuvre du Français Paul Andreu, se présente comme un vaisseau spatial de forme ovoïde ; situé à proximité de la place Tien An Men, il rappelle les ambitions culturelles de la Chine.

Ces cinq œuvres architecturales réalisées par des concepteurs de renommée mondiale pour souligner l'internationalisme de la ville ne doivent cependant pas cacher les multiples chantiers de rénovation qui modifient les formes de la ville. Ces chantiers sont généralement dirigés par des architectes chinois autour de centres commerciaux (Twin Tower, The Place, Lane Crawford...), de nouvelles résidences, d'hôtels et de centres d'affaires. Ces architectes, souvent formés à l'étranger, tentent de proposer un urbanisme chinois haut de gamme qui pourrait répondre aux questions de gestion du patrimoine, de lutte contre la pollution, d'ultra densité des villes, de transports urbains et qui pourrait s'exporter dans d'autres villes des cinq continents. La ville change de forme et certains auteurs considèrent que les espaces destinés aux bureaux, aux commerces et aux logements correspondent à l'équivalent de trois Manhattan. La construction d'autoroutes, de pénétrantes, l'extension des lignes de monorails et de métros, l'amélioration des rues et des espaces verts de la cité complètent le dispositif et, par leur rapidité, sont sans précédent dans les mutations urbaines.

Ces mutations ambitieuses ont un prix sur le plan social : une grande partie du patrimoine historique est détruit, plus de 300000 résidents du centre ville sont délogés et leurs maisons rasées, de nombreux réseaux sociaux de vie communautaire sont détruits... Le passage accéléré à une économie de marché, contrôlée en partie seulement par l'État, s'accompagne du renforcement d'inégalités sociales, de spéculations et de corruptions. La façade clinquante des jeux ne cache pas les dérives qui éloignent le pays d'une vision égalitaire du socialisme. Ces points noirs sont épinglés par le Collectif anti-Jeux olympique (CAJO) qui rappelle que la Chine reste une dictature qui exécute ses opposants sur la place publique et qui pratique la torture, l'enfermement arbitraire, la répression et la persécution du peuple tibétain. II affirme : «le $\mathrm{ClO}$ ne peut que se réjouir de l'organisation de ses jeux dans un état-prison ; il pourra ainsi déverser dans ce nouvel eldorado économique la propagande des multinationales qui le sponsorisent avec la complicité des forces armées ». Les manifestations dénonçant les atteintes aux droits des personnes lors du passage de la flamme olympique, notamment à Londres et Paris, au début de l'année 2008, confirment l'opposition de groupes organisés à la célébration des jeux en Chine.

Ces observations et ces actions ont cependant peu d'effets sur l'impact du projet et, notamment, sur ses visées médiatiques et touristiques. Outre la ville de Pékin, les JO sont l'occasion de valoriser le tourisme à l'ensemble du pays, aux zones littorales et en particulier à la ville de Qingdao dans la province du Shandong, qui organise les épreuves nautiques. Située à mi-distance de Pékin et de Shanghai, la ville (deuxième port de Chine et quatorzième port mondial) est aussi une station balnéaire réputée pour ses paysages côtiers ; elle dispose de trois parcours de golf professionnels, de 51 hôtels de trois étoiles et plus, et elle reçoit déjà près de 17 millions 
de touristes chinois et étrangers. Les JO sont l'occasion d'en faire une des premières villes de tourisme littoral d'Asie, avec un port de bateaux de croisières, un centre de conférences internationales, un yacht-club et une série de marinas et de plages. Ainsi, les jeux de Pékin s'inscrivent dans la mutation d'un pays qui, après avoir réussi une percée dans les industries de production, vise à jouer dans la cour des grands pour renforcer son attractivité touristique, ses industries culturelles et amorcer son ouverture vers une société postindustrielle.

Les JO sont l'événement spatial par excellence puisqu'ils focalisent sur un lieu déterminé à l'avance l'attention du reste du monde. Cette combinaison d'unité de lieu, d'unité de temps et d'unité d'action offre à la ville et au pays choisis une visibilité qu'aucun autre événement n'est susceptible de proposer. Le site olympique devient, grâce au système médiatique mondial, le théâtre où se jouent la mise en scène d'un monde apparemment plus équitable et plus ordonné et la mise en image des performances et de l'excellence (Bolle de Bal et Vésir, 1999). On comprend mieux la valorisation de ce site d'enchantement dans un monde désenchanté. Le choix de la ville olympique est ainsi devenu la première compétition et, après une période où la décision dépendait souvent de la volonté du président du $\mathrm{ClO}$ ou de ses proches, la désignation résulte aujourd'hui d'un véritable affrontement géopolitique (Augustin et Gillon, 1994). Cette désignation reste une des principales missions des membres du ClO qui, après la présentation des dossiers retenus, se prononcent, à bulletins secrets, huit ans avant l'ouverture des prochains jeux.

Une fois désignée, la ville est contrainte d'édifier les équipements et les installations nécessaires au bon déroulement des épreuves. Cette contrainte de temps est l'occasion d'élargir le programme sportif en projet de ville ou en projet urbain, en proposant des infrastructures d'accueil, de transport et de communication qui participent à une accélération des mutations urbaines et à une valorisation de l'image de la ville. Les discours promotionnels qui accompagnent la reconnaissance géopolitique du site et de la nation choisis sont dominants et une seule capitale, Denver, a refusé les Jeux d'hiver qui lui étaient offerts après le vote négatif d'un référendum local. Le coût d'organisation est en effet très lourd pour la ville et le pays organisateur, malgré les aides et les retombées attendues. Mais les résultats urbanistiques et touristiques sont parfois à la hauteur des moyens mis en œuvre (comme dans le cas de Barcelone). Le débat concernant l'efficacité économique et urbaine reste cependant ouvert et certains ont évoqué le " complexe de Cendrillon » pour rappeler que, une fois la fête terminée, la ville ne conserve pas toujours les atouts de sa splendeur (Poncet, 2001). Au-delà des stades démontables, des constructions éphémères, des paquebots de croisière maintenus à quai pour l'hébergement, le prix exorbitant des jeux ne s'inscrit pas toujours dans des perspectives durables. La tendance des aménagements olympiques s'oriente cependant dans des visées à long terme et certains projets proposés lors des candidatures sont mis en œuvre, même si la ville n'est pas choisie. Les effets attendus ne sont d'ailleurs pas seulement urbanistiques, ils sont politiques, culturels et s'inscrivent de plus en plus dans des visées touristiques qui s'accompagnent du renforcement de la fréquentation des lieux et d'un attrait nouveau pour les installations urbaines. Le tourisme et les loisirs ont maintenant envahi la ville et lui confèrent un supplément d'urbanité (Duhamel et Knafou, 2007).

La course aux anneaux d'or reste cependant réservée aux villes riches, susceptibles de mettre en œuvre les installations nécessaires et d'assurer la sécurité des jeux. Les grandes métropoles des pays du sud qui connaissent les croissances les plus fortes et de sérieux problèmes d'organisation urbaine sont de fait mises hors-jeu de la compétition.

Jean-Pierre Augustin est professeur à l'université de Bordeaux et chercheur à I'UMR ADES (Aménagement, Développement, Environnement, Santé et Sociétés) du Centre national de la recherche scientifique.

\section{Bibliographie}

Augustin, Jean-Pierre (2007), Géographie du sport, spatialités contemporaines et mondialisation, Paris, Armand Colin.

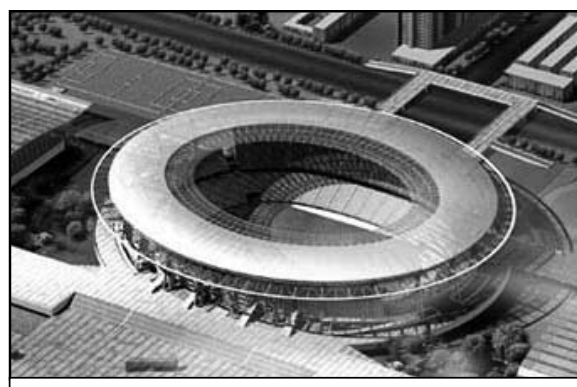

Illustration 4 : Stade olympique des jeux de Beijing présenté en image 3D. Source : @ BOCOG.

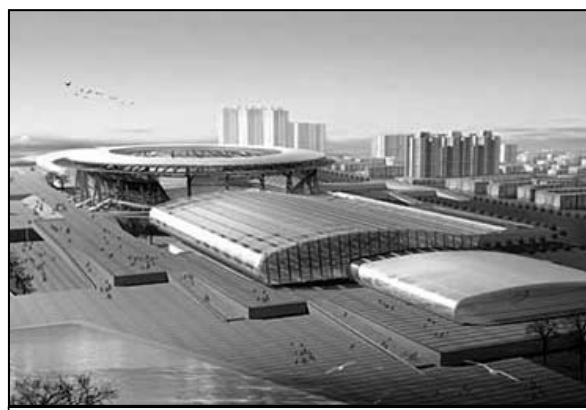

Illustration 5 : Complexe olympique des jeux de Beijing présenté en image 3D.

Source : @ BOCOG.

Augustin, Jean-Pierre et Pascal Gillon (2004), L'olympisme, bilan et enjeux géopolitiques, Paris, Armand Colin

Bolle de Bal, Marcel et Dominique Vésir (1999), Le sportif et le sociologue. Sport, individu et société, Paris, L'Harmattan.

Devillers, Christian (1994), Le projet urbain. Conférence, Paris, Pavillon de l'Arsenal.

Duhamel, Philippe et Rémy Knafou (2007), Mondes urbains du tourisme, Paris, Belin.

Huet, Bernard (1993), "Le hasard et la nécessité sont la loi du projet urbain. Entretien avec F. Edelmann ", Le Monde, 23 novembre, p. 2.

Gold, J. et M.M. Gold (2007), Olympic Cities: City Agendas, Planning, and the World's Games, 1896 to 2012, Londres, Routledge.

Poncet, P.P. (2001), « Sydney et les JO ou le complexe de Cendrillon ", Pouvoirs locaux, no 49 p. 78-81.

Seguillinas, Manuel (1993), "Barcelone, ou le poids des grands événements et des Jeux olympiques de 1992 dans la modernisation d'une métropole ", Hommes et terres du Nord, no 2, p. 104-109.

Sobry, Claude (1993), « Les grandes manifestations et installations sportives prétextes à l'aménagement du territoire ", Hommes et terres du Nord, $n^{\circ} 2$, p. 69-73.

Ville de Montréal (1991), Étude sur les incidences économiques et sociales du pôle "Parc olympique-Jardin botanique " sur le quartier Maisonneuve, rapport final, Montréal. 